\title{
AODV Routing Analysis On Multi-Hop Mobile AD HOC Network Connection
}

\author{
Yulita Salim
}

\begin{abstract}
The purpose of this study was to analyze the Mobile Ad-Hoc Network (MANET) connection on the multi-hop path using the $A O D V$ routing algorithm. MANET is formed from a set of nodes that are connected to the wireless interface. Parameters for measuring the simulated throughput are transmission range, simulation time, and topology size, number of Ad hoc nodes, source number, gateway number, Traffic Type, Rate Package, Package Size, and Pause Time. The result of simulation analysis of $A O D V$ routing algorithm is packet delay sent by the random average of 3.00088011 s with packet size 48 byte, throughput equal to 127,962459 bps. Packet loss depends on the field conditions at the time of simulation.
\end{abstract}

Keywords: MANET; AODV Routing; Wireless Network; Throughput.

\section{INTRODUCTION}

The MANET is required in situations where the communications infrastructure remains unavailable or damaged. Analytical research on MANET has examined the probability of two connected nodes resulting in a relationship pattern between node deployments with probabilities on single-hop and 2-hop connections assuming that different paths are mutually free, and the location of movement $\mathrm{X}$ and $\mathrm{Y}$ coordinates are normally distributed [1]. In this research will analyze the performance of throughput or average data size received by unity of time $(\mathrm{kbps})$ based on routing mechanism with NS with network topology made for Wireless Ad Hoc network [2]

\section{EXPERIMENTAL DETAILS}

Ad hoc mobile network is a multi-hop wireless network comprising a collection of mobile nodes that are dynamic and spontaneous and can be applied anywhere without using the network infrastructure [3]. Each node on an Ad Hoc network must be able to maintain the performance of data packets traffic in the network due to the mobility of nodes by way of network reconfiguration. For example, if there is a shifted node that results in a network outage, the node that is experiencing the interference may request the establishment of a new link route to forwarding packet data transmission [4]. The Ad-hoc On-Demand Distance Vector (AODV) routing algorithm is a routing algorithm that most widely studied in the Ad-Hoc environment [5]. Bjorn Wiberg and Erik Nordstrom from Uppsala University, Sweden, at the end of 2002 have successfully developed AODV-based routing protocol and are named AODV-Act.

Revised Manuscript Received on April 19, 2019.

Yulita Salim, Faculty of Computer Science, Universitas Muslim Indonesia, Makassar, Indonesia. (Email: yulita.salim@umi.ac.id)
This protocol can be used in real environments (using multiple laptops or mobile phones) or on simulations. AODV only uses symmetric links because the route request packets follow the route back from the route request packet. The advantage of AODV is that this protocol does not create additional traffic for communication on existing links [6]. In addition, distance vector routing is quite simple and does not require much memory allocation for routing calculations Based on the survey the authors set some parameters to measure the output (throughput) simulation [7]. These parameters are transmission range, simulation time, topology size, number of Ad Hoc nodes, Number of Sources, Number of Gateway, Traffic Type, Rate Package, Package Size, and Pause Time. The analysis technique from the simulation result is as follows: a) Design a MANET network simulation using AODV Algorithm on Network Simulator (NS). b) Test the results of the design, so that will be formed files that can be analysed to obtain network throughput. c) Simulation results using throughput parameters, packet drop, bottleneck, package queue, and delay are analysed.

\section{RESULT AND DISCUSSION}

The parameters measured in the simulation are transmission range, simulation time, topology size, number of Ad Hoc nodes, Number of Sources, Number of Gateways, Traffic Type, Rate Package, Package Size, and Pause Time. Based on the result of simulation design using AODV Algorithm its value respectively in the Table 1 .

Table 1: Parameter of simulation

\begin{tabular}{lc}
\hline Parameter & Value \\
\hline Transmission & $250 \mathrm{~m}$ \\
Reach & \\
Simulation Time & $30 \mathrm{sec}$ \\
Topology Size & $500 \times 500$ \\
& $\mathrm{~m}$ \\
Nodes of Ad Hoc & 3 \\
Number of Source & 2 \\
Number $\quad$ of & 1 \\
Gateways & TCP, \\
Type of Traffic & UDP \\
Rate Package & 4 \\
& packets/sec \\
Package Size & $85.8 \mathrm{Mb}$ \\
\hline
\end{tabular}


International Conference on Recents Advancements in Engineering and Technology (ICRAET-18) |15th and 16th March 2019|Siddhartha Institute of Technology \& Sciences, Telangana, India

based items, release inconveniences and immense confirmation of foul oil is imported from explicit worldwide areas which control the greater oil fields. Because of the ones reasons analysts tackle trade gas resources, so vegetable fuel research end up being force among explicit investigationsEase of UseThere are three numbers of nodes in the simulation, is node 0 , node 1 , and node 2 . To evaluate the performance of the AODV algorithm on Ad Hoc network, several simulations are performed. The simulation scenario can be seen in Figure 1.

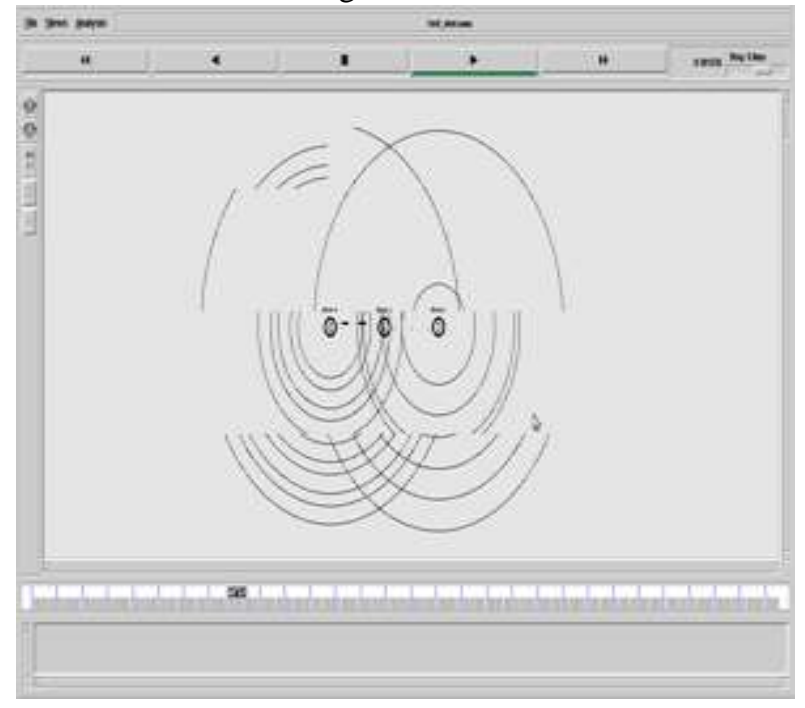

Fig. 1: Output simulation 2 hops 8.101 sec.

In Figure 1 looks 3 pieces of nodes. The time to start packet data transmission is in the first ten seconds of simulation. After that, each node will run on the coordinates that have been set in the script up to the specified range. The simulation stops at 30 seconds at a certain distance (less than $250 \mathrm{~m}$ ) will show packet loss. In NS each event will be recorded in a file with extension.tr. trace graph file results are large enough capacity. The simulation has been set and lasts for 30 seconds with $2.0 \mathrm{~ms}$. step.

\subsection{Analysis of delay}

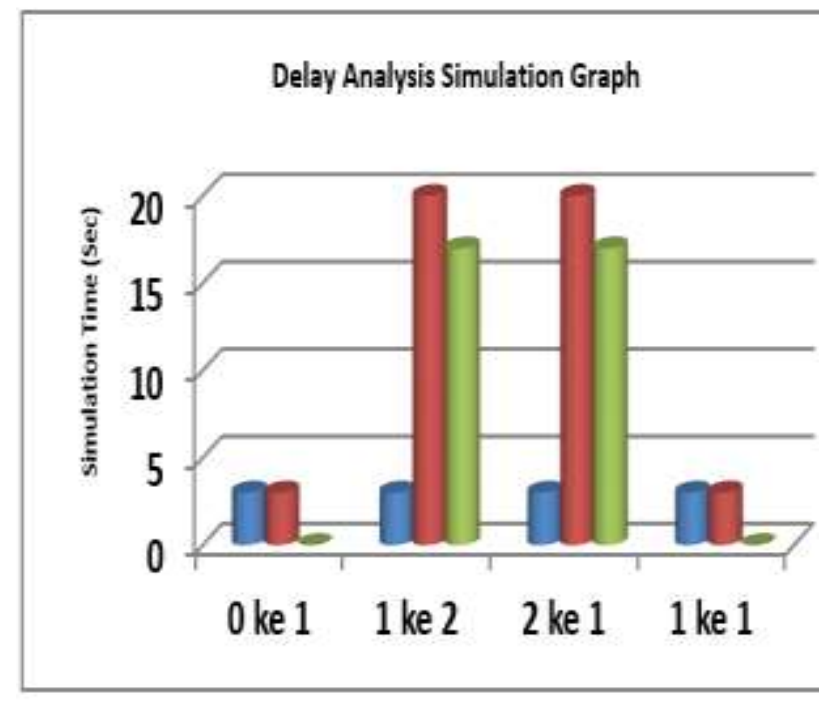

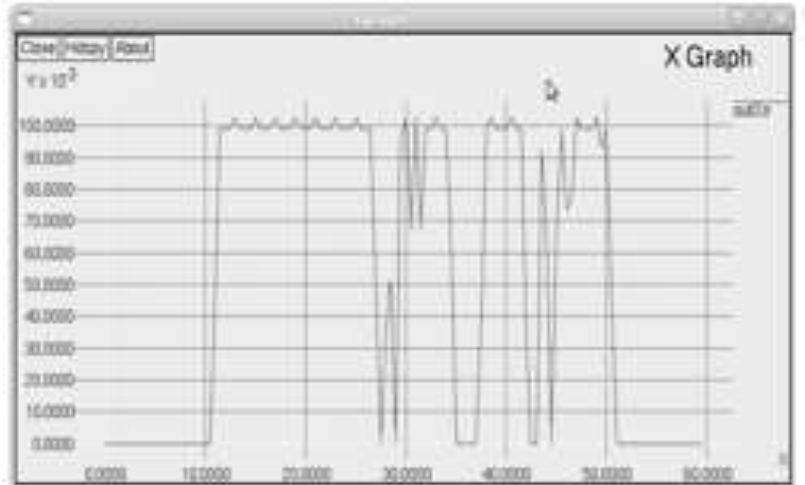

Fig. 2: Graph of Delay 2-Hop Analysis of AODV routing

Data Delay Time Packet can be seen in the Figure 2 . Graph of throughput of AODV 2-hop Ad-Hoc simulation. Packages are sent simultaneously from node 0 and node 1 at the 3 rd second in the simulation. Eg from node0 to node1 send time is at seconds to 3.0009630 and the time the packet is received is 3.0018692 so the delay is $0.0009062 \mathrm{~s}$. In this node 1 the process of routing occurs before the packet is sent back, in the case of the running routing algorithm is AODV reactive routing (based on the routing set in the simulation program). Delay obtained in this process is $0.0020107 \mathrm{~s}$. The data delay obtained varies, this is due to the movement of each sending node (source) and the destination node.

\subsection{Analysis throughput}

Throughput Calculation is the number of data packets received by mobile station divided by time of data transmission the mobile station. The plotted graph displays the $\mathrm{x}$-axis and $\mathrm{y}$-axis. The $\mathrm{x}$-axis represents the time and $\mathrm{y}$ axis describing the simulated throughput. Figure 2 shows an image released by the NS simulation software based on data included in the simulation script on Ad-Hoc Single-Hop network simulation [8]. The packet data transmission starts at $10 \mathrm{~ms}$. Until the 28th time throughput still shows a good data rate that is at $100 \mathrm{kbps}$ point. Figure 3. is the result of simulation that is caused by changes in movement and location of the mobile node to the base station in a network. Delay time indicates that there is a distance and there is movement in a cell base station. From the trace file, an Id packet that is randomly sent has an average delay of $3,00088011 \mathrm{~s}$ with the packet size of 48 bytes, obtained throughput of $127.962459 \mathrm{bps}$.

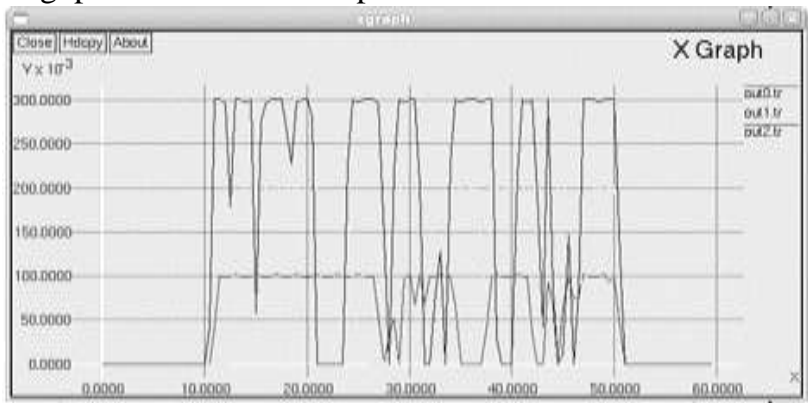

Fig. 3: Graph of throughput of AODV 2-hop Ad-Hoc simulation 


\section{CONCLUSION}

In Ad Hoc network simulations, the time for data transmission varies. This is influenced by the location and obstacle factors or obstacles that exist around the node that will exchange data packets on packet delivery. From the trace file, where an Id packet is sent at random, has an average delay of $3,00088011 \mathrm{~s}$ with the packet size of 48 bytes, found throughput of $127.962459 \mathrm{bps}$

\section{REFERENCES}

1. S. Mahapatro, P. K. Sahu, and P. K. Dash, "A hybrid active queue management technique for QoS based unicasting protocols in MANETs," Int. J. Eng. Technol., vol. 7, no. 2.6, pp. 58-63, 2018.

2. R. Kumar and R. Rishi, "Routing and security analysis in vehicular ad-hoc networks (VANETs)," in 2016 IEEE 1st International Conference on Power Electronics, Intelligent Control and Energy Systems, 2016, pp. 1-5.

3. [3] R. Farhan and S. Ahmeda, "Routing Protocol for Mobile Ad Hoc Network," J. Adv. Comput. Netw., vol. 1, no. 3, 2013.

4. P. V. Patel and B. Kadhiwala, "Broadcasting techniques for route discovery in mobile Adhoc network-A survey," in 2016 3rd International Conference on Computing for Sustainable Global Development, 2016, pp. 671-674.

5. K. T. Ayanwuyi, M. F. Zuhairi, C. L. Zheng, and I. S. . Alias, "Simulation Technique of Steady-State Network based on AODV Routing Protocol," Int. J. Eng. Technol., vol. 7, no. 4.29, pp. 140-147, 2018.

6. M. K. Suthar, "Performance Analysis of AODV \& DSR Routing Protocol for Wireless Ad-hoc Network," Int. J. Res. Appl. Sci. Eng. Technol., vol. 3, no. 2, pp. 245-251, 2015.

7. V. Matre and R. Karandikar, "Multipath routing protocol for mobile adhoc networks," in 016 Symposium on Colossal Data Analysis and Networking, 2016, pp. 1-5.

8. A. Dorri, S. R. Kamel, and E. Kheyrkhah, "SECURITY CHALLENGES IN MOBILEAD HOC NETWORKS:ASURVEY," Int. J. Comput. Sci. Eng. Surv., vol. 6, no. 1, pp. 15-29, 2016 